Pramāṇa -J. Phys., Vol. 25, No. 2, August 1985, pp. 223-224. (C) Printed in India.

\title{
Some remarks on a model for internal structure of black holes
}

\author{
P GOSWAMI and K P SINHA \\ Division of Physics and Mathematical Sciences, Indian Institute of Science, \\ Bangalore 560012, India \\ MS received 22 May 1985
}

Abstract. Some clarifications about a model for the internal structure of black holes developed in an earlier paper are presented to remove confusion in the interpretation of certain results.

Keywords. Black holes; thermodynamics; internal structure; binding energy.

In a recent paper, we developed a model for the internal structure of black holes which could account for the thermodynamic relations for black holes obtained by Hawking (Goswami and Sinha 1984 hereafter referred to as GS). In this model a black hole was treated as an assembly of planckions (with mass $M_{P}=\left(\hbar c / 4 G_{N}\right)^{1 / 2}, \hbar, c, G_{N}$ being the universal constants) having fermionic character. This model is a representation of a black hole in terms of planckions and one must bear this in mind while interpreting the results. Thus, for example, although in this representation a black hole is a super dense object of planckions, it does not mean that the actual matter density will also become necessarily very high. (It is well known that the radius of the observable universe $\sim 10^{28} \mathrm{~cm}$ with a critical density $\rho_{\mathrm{c}} \sim 10^{-29} \mathrm{~g} / \mathrm{cm}^{3}$ would satisfy the mass-radius relation for a Schwarzschild black hole).

In the planckionic picture introduced by us the 'observed' mass of a black hole should be defined as

$$
M_{B}=M_{N}-B
$$

where $M_{N}$ is the nucleonic mass of the object and $B$ is the binding energy. By nucleonic mass we mean the total sum of the rest masses of the constituents (here planckions) when they are dispersed to infinity. The binding energy is the energy that must be supplied to disperse the object tainfinity (see Narlikar 1978). The nucleonic mass $M_{N}$ in the present case is given by

$$
M_{N}=N_{P} M_{P},
$$

where $N_{P}$ is the total number of planckions in the volume $V_{B}$ of the black hole and is given by (cf. equation (3) of Gs)

$$
N_{P}=\frac{V_{B} g}{6 \pi^{2}}\left(\frac{c^{3}}{\hbar G_{N}}\right)^{3 / 2} \text {. }
$$


Thus

$$
M_{N} \sim \frac{g}{6 \pi^{2}}\left(\frac{V_{B}}{L_{P}{ }^{3}}\right) M_{P},
$$

where $L_{P}=\left(\hbar G_{N} / c^{3}\right)^{1 / 2}$ is the planck length. Also it is easy to see that the rest mass of two planckions which are within planck length to each other is nearly consumed by their gravitational energy. Similarly it can be shown that for a black hole of volume $V_{B}$ $\sim 10^{16} \mathrm{~cm}^{3}$ (which corresponds to a black hole of solar mass) the binding energy of the constituents is of the order of nucleonic mass $\left(\sim 10^{109} \mathrm{~g}\right)$. Thus the smallness of $M_{B}$ (in this case $M_{\odot} \sim 10^{33} \mathrm{~g}$ ) is understandable.

Further, our expression relating the degeneracy pressure $P_{0}$ with the gravitational interaction of the black hole is not equivalent to the conventional one. Here we are dealing with a black hole of mass $M_{B}$ interacting with its nucleonic mass $M_{N}$ with

$$
P_{G} \approx \frac{M_{B}}{R_{0}} \frac{M_{N}}{V_{B}}
$$

and degeneracy pressure

$$
P_{0} \approx \frac{1}{4}\left(6 \pi^{2} / g\right)^{1 / 3} \hbar c n_{P}^{4 / 3},
$$

$n_{P}$ being the number density of planckions. The effective mass $\left(M_{B}\right)$ of the black hole in the planckionic representation lies on the surface with the interior mass of planckions being consumed by gravitational interactions. The above approach is a prescription to yield the 'equilibrium' radius of a black hole. However, this argument is applicable to cases where $2 m c^{2} \sim G m^{2} / r_{0}$, where $m$ is the mass of the constituent particles and $r_{0}$ is the characteristic distance. Thus for ordinary stellar objects or neutron stars the above argument should not be applied.

Finally it should be remarked that once the black hole radius $R_{0}=2 G M / c^{2}$ is known, other relations for black hole temperature and entropy follow as in our paper. In fact one could have straightaway used the Schwarzchild radius and carried out calculations using the planckionic representation of a black hole consisting of a relativistic gas of fermions.

\section{References}

Goswami P and Sinha K P 1984 Pramana 23381

Narlikar J V 1978 General relativity and cosmology (Bombay: Macmillans) 